\title{
Coupling CRISPR to smart hydrogels
}

The CRISPR-Cas system has become a powerful tool in genome editing and now expands its footprint into materials science.

S mart hydrogels, those that can change their properties following a biologically relevant cue, have numerous biomedical applications, ranging from drug release to biosensing. Such responsiveness typically involves the synthesis of a hydrogel matrix.

For example, DNA-responsive hydrogels rely on the incorporation of specific DNA fragments that serve as structural elements and can respond to an external stimulus via, for example, strand-displacement. To reprogram the responsiveness, researchers often have to re-design and synthesize entirely new hydrogels.

James Collins, a bioengineer from MIT, and his team hope to develop a 'plug-andplay' approach that can reprogram the responsiveness by simply redesigning the 'cue' unit. They recognized the potential of CRISPR-Cas enzymes in regulating the properties of DNA-based material. Collins comments, "these nucleases are inherently programmable and are capable of both recognizing user-defined DNA sequences and cleaving them enzymatically."

Collins and colleagues explored the possibility of using Cas enzymes to actuate DNA-hydrogels that can respond to CasgRNA activation and thus change hydrogel properties through user-defined input materials, including Cas-gRNA complexes and dsDNA triggers. Among the options of available Cas enzymes, they chose Cas12a for its collateral activity in both targeted dsDNA and non-specific ssDNA cleavages. Collins also notes that recently described Cas14a "could similarly be used to cleave DNA elements present in smart materials to further expand the range of targets of CRISPR-actuated materials."

The non-specific activity of Cas12a couples one dsDNA binding event to multiple ssDNA cleavages, thus allowing for an amplified transduction from external stimulus to changes in hydrogel properties. This is in contrast to the traditional stranddisplacement reaction that usually exhibits a one-to-one stoichiometry of trigger DNA to crosslinker DNA. Moreover, the ssDNA degradation rate depends on the amount and sequence of trigger dsDNA, thus

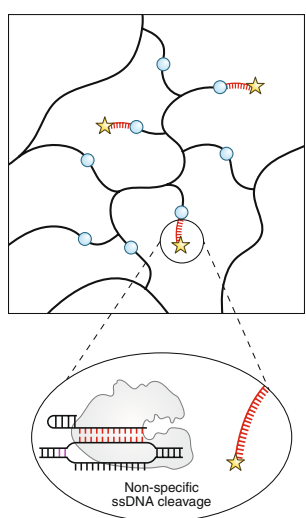

In DNA-hydrogels, Cas12a binds and cleaves target dsDNA, as well as non-specifically degrading ssDNA nearby.

offering a way to manipulate the dynamics of how DNA-hydrogels respond.

To demonstrate the versatility of this approach, they showcased three CRISPRhydrogel systems, including PEG-DNA hydrogels for release of DNA-anchored compounds, polyacrylamide-DNA hydrogel for release of preloaded cargos such as live cells, and carbon-back-DNA hydrogels used as an electrical fuse.

Despite this versatility, it is not a perfect system. For a given set of gRNAs designed to recognize different DNA sequences associated with a single target (for example, a gene or genome), it is still a challenge to predict from sequence alone which will lead to more efficient activation of the Cas enzyme.

Looking forward, the Collins lab is excited about the developments in low-cost biosensors for use in disease outbreaks and applications in tools, for example, to perturb the extracellular environment of cells through releasing specific adhesion sites on demand.

\section{Lei Tang}

Published online: 27 September 2019 https://doi.org/10.1038/s41592-019-0609-2

\section{Research paper}

English, M. A. et al. Programmable CRISPR-responsive smart materials. Science 365, 780-785 (2019). nature MASTERCLASSES

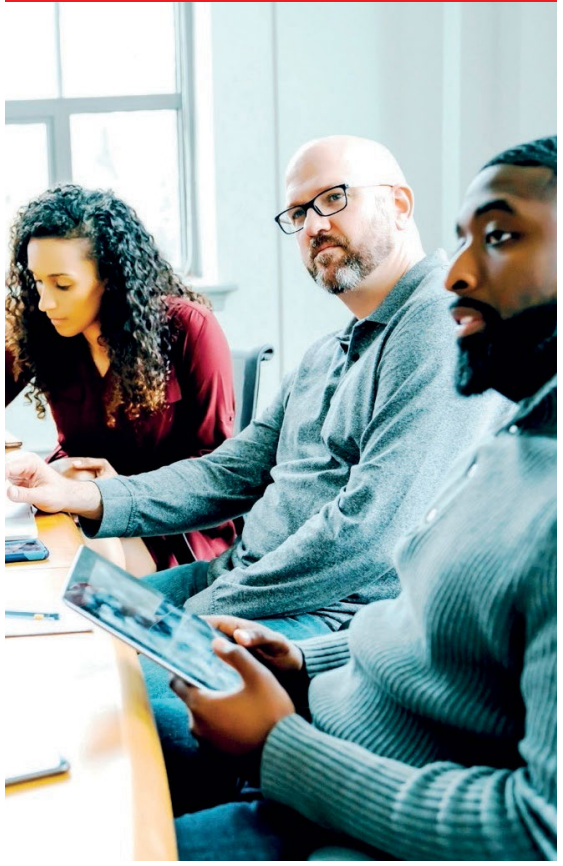

Workshops in

Scientific Writing

and Publishing

Delivered by Nature Research

journal editors and hosted

at institutions worldwide,

researchers learn how to

turn great science into great

papers.

\section{Find out more at \\ masterclasses.nature.com}

W masterclasses.nature.com

in Follow us on Linkedln 